\title{
Correction to: A low order dynamical model for runoff predictability
}

\author{
Roman Olson ${ }^{1,2,3}$ (I) Axel Timmermann ${ }^{2,3} \cdot$ June-Yi Lee ${ }^{2,4} \cdot$ Soon-II An ${ }^{1,5}$
}

Published online: 6 May 2021

(c) The Author(s) 2021

\section{Correction to: Climate Dynamics (2021) 56:399-422 \\ https://doi.org/10.1007/s00382-020-05479-w}

In the original publication of the article, a typo error has been occurred in an institution name. Specifically Center for Climate Physics, Institute for Basic Research, Busan, South Korea should be corrected to Center for Climate Physics, Institute for Basic Science, Busan, South Korea.

The original article has been corrected.

Publisher's Note Springer Nature remains neutral with regard to jurisdictional claims in published maps and institutional affiliations.

The original article can be found online at https://doi.org/10.1007/ s00382-020-05479-w.

Roman Olson

romanolson@yonsei.ac.kr

1 Irreversible Climate Change Research Center, Yonsei University, Seoul, South Korea

2 Center for Climate Physics, Institute for Basic Science, Busan, South Korea

3 Pusan National University, Busan, South Korea

4 Research Center for Climate Sciences, Pusan National University, Busan, South Korea

5 Department of Atmospheric Sciences, Yonsei University, Seoul, South Korea 